Draft VERSION NOVEMBER 22, 2021

Typeset using LATEX default style in AASTeX63

\title{
Geometrical and Kinematic Parameters of the Jet of the Blazar S5 0716+71 in a Helical-Jet Model
}

\author{
Marina S. Butuzova (iD 1 \\ ${ }^{1}$ Crimean Astrophysical Observatory of RAS, 298409, Nauchny, Russia
}

(Published Astronomy Reports, Volume 62, Issue 2, pp.116-122, 2018, DOI: 10.1134/S1063772918020038)

\begin{abstract}
Periodic variations of the position angle of the inner jet of the blazar S5 0716+71 suggest a helical structure for the jet. The geometrical parameters of a model helical jet are determined. It is shown that, when the trajectories of the jet components are non-ballistic, the angle between their velocity vectors and the line of sight lies in a broader interval than is the case for ballistic motions of the components, in agreement with available estimates. The contradictory results for the apparent speeds of components in the inner and outer jet at epochs 2004 and 2008-2010 can be explained in such a model. The ratio of the apparent speeds in the inner and outer jet is used to derive a lower limit for the true speed of the components $(\beta>0.999)$ and to determine the pitch angle of the helical jet $\left(p=5.5^{\circ}\right)$. The derived parameters can give rise to the conditions required to observe high speeds (right to $37 c$ ) for individual jet components.
\end{abstract}

Keywords: blazar, S5 0716+714, helical jet

\section{INTRODUCTION}

The BL Lac object S5 $0716+71$ is a highly variable source on time scales of both about a day (Montagni et al. 2006; Poon et al. 2009; Vol'Vach et al. 2009; Gorshkov et al. 2011a,b) and several years (Dai et al. 2013; Volvach et al. 2012; Bychkova et al. 2015). Its redshift is not precisely known; estimates of $z$ based on observations of nearby galaxies range from $z=0.26$ to $z \geq 0.52$ (Wagner et al. 1996; Bychkova et al. 2006; Sbarufatti et al. 2005). It has been intensely observed over the entire electromagnetic spectrum (see, e.g. Wagner et al. 1996; Liao et al. 2014). Very Long Baseline Interferometry (VLBI) observations of S5 0716+71 have been conducted over more than 10 years (Bach et al. 2005; Britzen et al. 2009; Rani et al. 2015), for example as part of the MOJAVE program (Lister et al. 2013). VLBI observations at 5, 8.4, 15, and $22 \mathrm{GHz}$ during 1992.74-2001.2 revealed variations in the position angle of the inner jet at distances up to 1 milliarcsecond (mas) from the core with a period of $7.4 \pm 1.5$ yrs and an amplitude of $3.5^{\circ}$ (Bach et al. 2005). Analysis of $15 \mathrm{GHz}$ VLBI data for 1994.5 - 2011.5 showed the period of variations of the position angle of the inner part of the jet (closest to the core), $\mathrm{PA}_{\mathrm{in}}$, to be $10.9 \mathrm{yrs}$, with an amplitude of $11^{\circ}$ (Lister et al. 2013). Periodic variations of $\mathrm{PA}_{\text {in }}$ can be most simply explained if the jet has a helical shape (Bach et al. 2005; Lister et al. 2013).

This hypothesis has been widely applied in interpretations of the observed properties of the jets of active galactic nuclei (AGNs). For example, a helical jet shape arising due to precession of the central black hole has been used to explain the apparent motions of superluminal components and long-period brightness variations in the AGNs OJ 287 (Abraham 2000), BL Lac (Caproni et al. 2013), 3C 120 (Caproni \& Abraham 2004), and 3C 279 (Abraham \& Carrara 1998). The shape of the spectral energy distribution of the blazar Mrk 501 and its time variations have also been interpreted in terms of a helical jet model (Villata \& Raiteri 1999). A helical jet could form as a result of the development of magnetohydrodynamical instability arising in the jet flow (see,e.g., Hardee \& Norman 1988). Spiral structure could also form due to the twisting of a magnetic rope in and around a conical jet (Meier et al. 2001), and the

Corresponding author: Marina Butuzova

mbutuzova@craocrimea.ru 


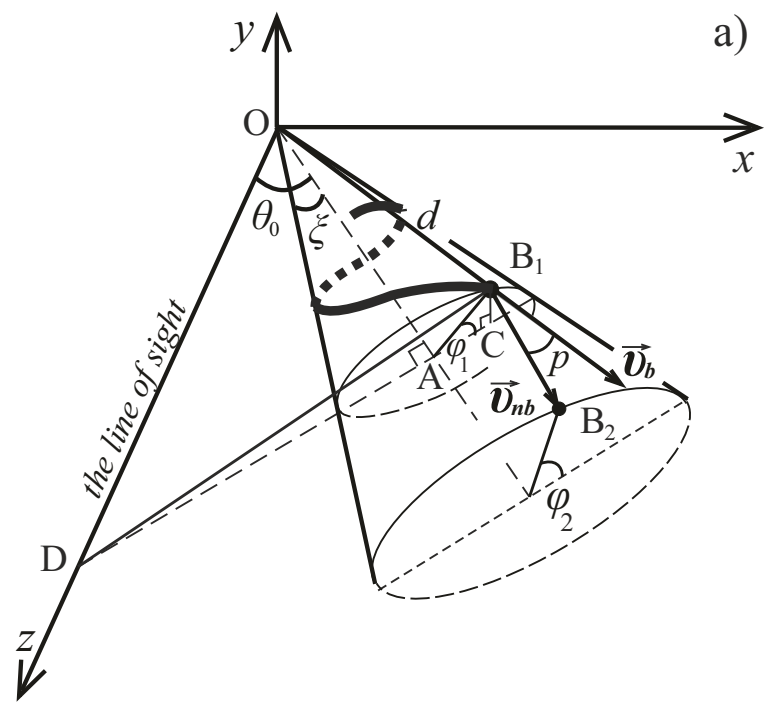

b)

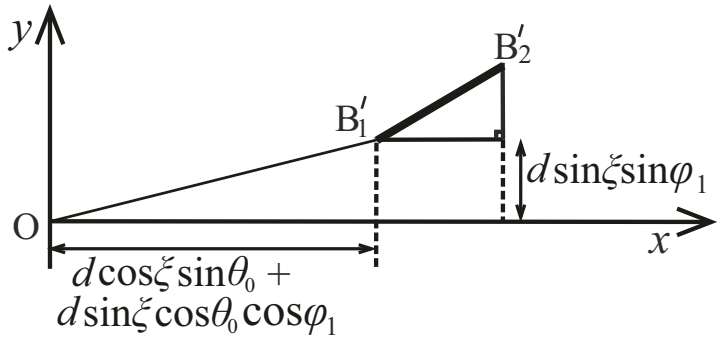

Figure 1. (a) Schematic of the geometrical parameters of a helical jet. The jet is shown by the bold curve (a section of the jet located on the opposite side of the cone is shown dotted). At the time shown in this figure, the component $\mathrm{B}_{1}$ is located a distance $d$ from the vertex of the cone. The velocity vectors for ballistic $\left(\boldsymbol{v}_{b}\right)$ and non-ballistic $\left(\boldsymbol{v}_{n b}\right)$ motions are shown. (b) Projection of the schematic (a) onto the celestial sphere. The segment $\mathrm{B}_{1}^{\prime} \mathrm{B}_{2}^{\prime}$ is the projection of the trajectory of a component moving with speed $\boldsymbol{v}_{n b}$ over a time $d t$.

plasma near a magnetized accretion disk follows helical trajectories (Camenzind \& Krockenberger 1992). Returning to the blazar S5 $0716+71$, it has been proposed that a shock moving along a curved, possibly helical, trajectory can provide a good explanation for the behavior of its gamma-ray, optical, and radio flares (Rani et al. 2015; Larionov et al. 2013; Rani et al. 2013).

In the study presented here, we have used the period and amplitude of variations of the inner-jet position angle $\mathrm{PA}_{\text {in }}$ (Lister et al. 2013) to derive the geometrical parameters of the helical curve formed by the jet components (Section 2). In Section 3, we use these parameters to derive intervals of possible values for the angle between the line of sight and the velocity vectors of the jet components for the cases of ballistic and non-ballistic motion. We show in Section 4 that it is possible to reconcile the different detected apparent component speeds for the inner and outer jet of S5 0716+71 presented by citetRastorgueva11 for epoch 2004 and by Rani et al. (2015) for epoch 2008-2010 only if the component motions are non-ballistic. In this case, the condition for high apparent jet-component speeds reaching $37 c$, as is observed for S5 $0716+71$ Rani et al. (2015), is realized. It is also possible to determine a lower limit on the true speed of the jet components. The main results of this study are summarized in Section 5 .

It is important that our conclusions do not depend on the processes leading to the helical shape of the jet or the physical nature of the jet components. The reasoning and conclusions presented here can be applied to various objects that we can treat as components of a jet. For example, if radiation is produced by the entire jet, a jet component could correspond to a volume element in the jet. If distinct parts of a stream radiate (plasmoids, shock fronts where electrons are accelerated and then radiate), we can also consider these regions as jet components, without specifying their nature.

\section{DETERMINATION OF THE GEOMETRICAL PARAMETERS OF A HELICAL JET}

Let us suppose that a jet component is located on the surface of a cone and follows a helical curve beginning at some distance from the vertex of the cone (Fig. 1a). The vertex of this cone may not coincide with the position of the central supermassive black hole or the position of the physical beginning of the jet. The latter probably has a parabolic shape, but the size of this region is substantially smaller than the distances considered in this study.

We introduce a coordinate system with its origin at the cone vertex. The $\mathrm{OZ}$ axis is directed along the line of sight and the OX axis along the projection of the cone axis OA onto the plane of the sky. The opening angle of the cone and the angle between the cone axis and the line of sight are denoted $\xi$ and $\theta_{0}$, respectively. All information about angles is given in degrees. Lister et al. (2013) determined $\mathrm{PA}_{\mathrm{in}}$ as the mean flux-density-weighted position angles of all the jet features located at distances of $0.15-1$ mas from the core. In contrast to this, we will simply consider the 


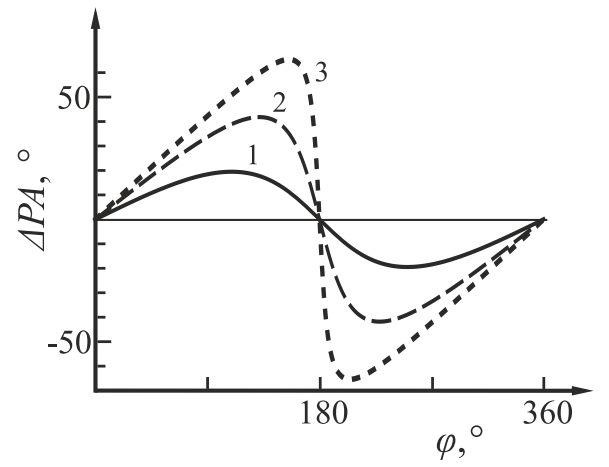

Figure 2. Theoretical deviation of the position angle of the inner jet from its mean value over one variation period for $\varphi$, for $\theta_{0} / \xi=3(1), 1.5(2)$, and $1.1(3)$.

position angles of components measured at the time when they reach a distance $d$ from the cone vertex. Note that the concept of the "inner jet" refers to that part of the jet located out to some distance from the VLBI core, usually specified in various studies to be $1-2$ mas. Another quantity specifying the position of a component on the surface of the cone is the azimuth angle $\varphi$, defined as the angle between the OXZ and $\mathrm{OAB}_{1}$ planes. As the jet material moves outward, the angle $\varphi$ for each successive component reaching the distance $d$ differs from that for the previous component, leading to variations in $\mathrm{PA}_{\text {in }}$. When $\theta_{0}<\xi$, the line of sight is inside the helical path formed by the jet. In this case, the inner-jet position angle $\mathrm{PA}_{\text {in }}$ varies by $360^{\circ}$ over the period of variations of $\varphi$, which does not agree with the observations of Bach et al. (2005); Lister et al. (2013). Therefore, we have restricted our consideration to the case $\theta_{0}<\xi$.

The mean position angle of the inner jet $\left(\mathrm{PA}_{0}\right)$ corresponds to the position of a component in the OXZ plane. Deviations of the measured values for $\mathrm{PA}_{\text {in }}$ from $\mathrm{PA}_{0}$, i.e., the angle between the $\mathrm{OX}$ axis and the radius vector of a component projected onto the plane of the sky $\left(\mathrm{OB}_{1}\right)$, is denoted $\triangle \mathrm{PA}$. As is shown in Fig. 1b, an expression for $\triangle \mathrm{PA}$ in terms of $\varphi, \theta_{0}$, and $\xi$ can straightforwardly be found from the projections of $\mathrm{OA}, \mathrm{OB}_{1}$, and $\mathrm{B}_{1} \mathrm{C}$ onto the plane of the sky:

$$
\tan \Delta P A=\frac{\sin \xi \sin \varphi}{\cos \xi \sin \theta_{0}+\sin \xi \cos \theta_{0} \cos \varphi} \approx \frac{\sin \varphi}{\theta_{0} / \xi+\cos \varphi},
$$

where we have approximated that the angles $\theta_{0}$ and $\xi$ are small. The observed position angle is then $\mathrm{PA}_{\text {in }}=\mathrm{PA}_{0}+\Delta \mathrm{PA}$. As can be seen from the right-hand side of (1), when $\theta_{0}$ and $\xi$ are small, $\mathrm{PA}_{\text {in }}$ depends on the ratio $\theta_{0} / \xi$. For definiteness, we will further assume that the helical jet is winding in the direction that leads to an increase in $\varphi$ as each successive component appears at the distance $d$. Figure 2 presents the variations of $\mathrm{PA}_{\text {in }}$ over one period calculated using the exact formula (1), for various values of the ratio $\theta_{0} / \xi$. As this ratio increases, the amplitude of the variations of $\mathrm{PA}_{\mathrm{in}}$ and the degree of asymmetry of the variation curve both decrease.

It follows from (1) that

$$
\varphi= \pm \arccos \left(-\tan \xi \cot \theta_{0}\right)
$$

and $\mathrm{PA}_{\text {in }}$ has maximum [+ in (2)] and minimum [- in (2)] values. Substituting (2) into (1) and making the substitution $\sin \varphi=\sqrt{1-\cos ^{2} \varphi}$ yields the amplitude of the variations of $\mathrm{PA}_{\text {in }}$ :

$$
\mathrm{PA}_{\max }=\arctan \left[\sin \xi\left(\cos ^{2} \xi \sin ^{2} \theta_{0}-\sin ^{2} \xi \cos ^{2} \theta_{0}\right)^{-1 / 2}\right] \approx\left[\left(\frac{\theta_{0}}{\xi}\right)^{2}-1\right]^{-1 / 2},
$$

which, like $\triangle \mathrm{PA}$, depends only on the ratio of $\theta_{0}$ and $\xi$ when these angles are small. To determine $\theta_{0} / \xi$ using (1), we used the value $\mathrm{PA}-\max =11^{\circ}$ (Lister et al. 2013), based on a more extensive series of observations with better angular resolution than in (Bach et al. 2005). Then,

$$
\theta_{0} / \xi \approx 5.3 .
$$

Various estimates of the angle of the jet of S5 $0716+71$ to the line of sight based on the observed apparent speeds of VLBI components have yielded $\theta_{0}$ values from $0.5^{\circ}$ to $12^{\circ}$ (Bach et al. 2005; Britzen et al. 2009; Rastorgueva et al. 


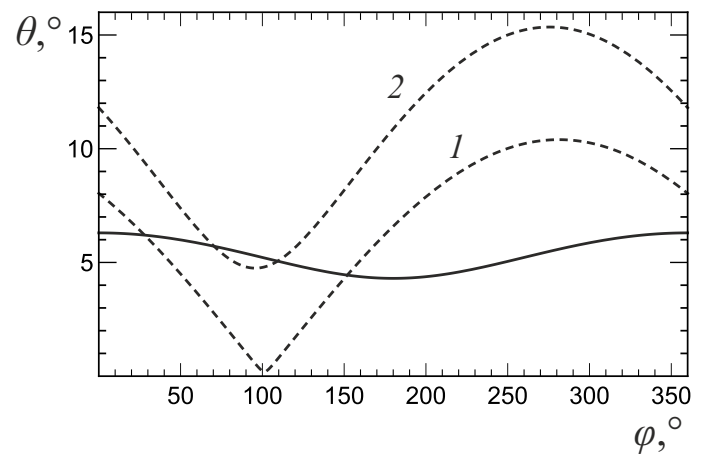

Figure 3. Dependence of the direction of the motion of jet components relative to the line of sight $(\theta)$ on the azimuthal angle $\varphi$ for ballistic motion (solid curve) and non-ballistic motion (dashed curves) with $p=5^{\circ}(1)$ and $p=10^{\circ}(2)$.

2011). However, these estimates were carried out using observations covering limited time intervals, and depend on the jet model considered. Therefore, we focused on the value obtained in (Pushkarev et al. 2009), where $\theta_{0}$ values were determined from the maximum apparent speeds of components displaying radial motion without acceleration, observed in the MOJAVE project over no fewer than five epochs. The value obtained for S5 0716+71 was $\theta_{0}=5.3^{\circ}$ (Pushkarev et al. 2009). A very similar value for $\theta_{0}$ was obtained by Savolainen et al. (2010). It follows from (4) that $\xi \approx 1^{\circ}$. Hence, the opening angle of the jet, that is, the angle inside of which jet components can be observed, is $2 \xi=2^{\circ}$. The opening angle deduced from analysis of VLBI maps of the blazar S5 0716+71 is $1.6^{\circ}$ (Pushkarev et al. 2009). Thus, we can see a good consistency between these estimates of the jet opening angle derived using independent methods.

\section{ANGLE BETWEEN THE VELOCITY VECTOR OF A JET COMPONENT AND THE LINE OF SIGHT}

In the case of ballistic motion, the velocity vector of a component $\left(\boldsymbol{v}_{b}\right)$ located a distance $d$ from the cone vertex is directed along the cone generatrix (Fig. 1). The angle $\theta_{b}$ between $\boldsymbol{v}_{b}$ and the line of sight can be found from the triangle $\mathrm{OB}_{1} \mathrm{D}$. The side $\mathrm{OD}$ can be found from the triangle $\mathrm{OAD}$ together with the relations $\angle \mathrm{DOA}=\theta_{0}$ and $\mathrm{OA}=d \cos \xi$, and the side $\mathrm{B}_{1} \mathrm{D}$ can be found from the triangle $\mathrm{AB}_{1} \mathrm{D}$ with the relations $\angle \mathrm{DAB}_{1}=180^{\circ}-\varphi, \mathrm{AB}_{1}=d \sin \xi$, $\mathrm{AD}=d \cos \xi \tan \theta_{0}$. The final expression for $\theta_{b}$ does not depend on $d$ :

$$
\cos \theta_{b}=\cos \xi \cos \theta_{0}-\sin \xi \sin \theta_{0} \cos \varphi .
$$

The value of $\theta_{b}$ decreases non-uniformly from $\theta_{0}+\xi=6.3^{\circ}$ to $\theta_{0}-\xi=4.3^{\circ}$ as $\varphi$ varies from $0 \circ$ to $180 \circ$, and grows symmetrically back to $6.3^{\circ}$ as $\varphi$ varies from $180^{\circ}$ to 360 。 (solid curve in Fig. 3).

In the case of non-ballistic motion, the velocity vector of a component is directed at a pitch angle $p$ to the cone generatrix. The angle between the line of sight and this velocity vector $v_{n b}$ can be obtained from the expression

$$
\sin \theta_{n b}=\mathrm{B}_{1}^{\prime} \mathrm{B}_{2}^{\prime} / \mathrm{B}_{1} \mathrm{~B}_{2}
$$

where $\mathrm{B}_{1}^{\prime} \mathrm{B}_{2}^{\prime}$ is the projection onto the plane of the sky of the segment $\mathrm{B}_{1} \mathrm{~B}_{2}=\left|v_{n b}\right| \cdot \Delta t$, traversed by the component over a time $\Delta t$. Figure $1 \mathrm{~b}$ presents the segment $\mathrm{B}_{1}^{\prime} \mathrm{B}_{2}^{\prime}$ together with the coordinates of the point $\mathrm{B}_{1}^{\prime}$. The coordinates of the point $\mathrm{B}_{2}^{\prime}$ can be found straightforwardly by replacing $\varphi_{1}$ with $\varphi_{2}$ and $d$ with $(d+\Delta d)$, where $\Delta d=v_{n b} \Delta t \cos p$ is the increase in the distance from the cone vertex to the component considered over a time $\Delta t$. As can be seen in Fig. $1 \mathrm{~b}, \mathrm{~B}_{1}^{\prime} \mathrm{B}_{2}^{\prime}$ can easily be found as

$$
\mathrm{B}_{1}^{\prime} \mathrm{B}_{2}^{\prime}=\sqrt{f_{a}^{2}+f_{b}^{2}}
$$

where

$$
\begin{gathered}
f_{a}=(d+\Delta d) \sin \xi \sin \varphi_{2}-d \sin \xi \sin \varphi_{1}, \\
f_{b}=(d+\Delta d)\left(\sin \xi \cos \theta_{0} \cos \varphi_{2}+\cos \xi \sin \theta_{0}\right)-d\left(\sin \xi \cos \theta_{0} \cos \varphi_{1}+\cos \xi \sin \theta_{0}\right),
\end{gathered}
$$

and $\varphi_{1}$ and $\varphi_{2}$ are the azimuth angles of the points $\mathrm{B}_{1}$ and $\mathrm{B}_{2}$. Knowing the change in the azimuth angle

$$
\Delta \varphi=\frac{v_{n b} \Delta t \sin p}{(d+\Delta d) \sin \xi}
$$


taking this to be small over the time $\Delta t$, and using the relation $\varphi_{2}=\varphi_{1}+\Delta \varphi$, the expression (6) acquires the form

$$
\sin \theta_{n b}=\sqrt{g_{a}^{2}+g_{b}^{2}}
$$

where

$$
\begin{gathered}
g_{a}=\cos p \sin \xi \sin \varphi_{1}+\sin p \cos \varphi_{1}, \\
g_{b}=\cos p\left(\cos \xi \sin \theta_{0}+\sin \xi \cos \theta_{0} \cos \varphi_{1}\right)-\sin p \cos \theta_{0} \sin \varphi_{1} .
\end{gathered}
$$

If $\varphi_{2}=\varphi_{1}-\Delta \varphi$, the signs of the terms $\sin p \cos \varphi_{1}$ and $\sin \xi \cos \theta_{0} \cos \varphi_{1}$ change. However, our conclusions do not depend on the sign of $\varphi_{2}-\varphi_{1}$.

Analyzing $\theta_{n b}(\varphi)$ using formulas (6) - (13) with various values of $p$, we found that this quantity lies in the interval $\theta_{n b} \approx p \pm 5^{\circ}$. Figure 3 shows the variations of $\theta_{n b}$ for the cases $p=5^{\circ}$ and $10^{\circ}$ (dashed curves).

\section{APPARENT AND PHYSICAL SPEEDS OF THE JET COMPONENTS IN S5 0716+71}

The results of different studies of the kinematics of the jet of the blazar S5 0716+71 are contradictory. Based on observations at 5, 8.4, 15, and $22 \mathrm{GHz}$ obtained in 1992.73-2001.17, Bach et al. (2005) report the presence of components moving rapidly in the radial direction (apparent speeds $\beta_{\text {app }}=5 c-16 c$ ), whereas more typical values for BL Lac objects are $\beta_{\text {app }} \leq 5 c$ (Gabuzda et al. 2000). On the other hand, observations from 1992.73-2006.32 did not exhibit any appreciable outward component motions, instead indicating the presence of stationary components (Britzen et al. 2009). That is, the jet components were located at roughly the same distances from the core during this entire interval, and any variations in their position angles were within the uncertainties. An analysis of data obtained at 43 and $86 \mathrm{GHz}$ during 2008 - 2010 indicated the presence of features in the inner jet (within 2 mas from the core) with apparent speeds up to $10 c$, which was lower than the values of $\beta_{\text {app }}$ detected farther from the core ( $\left.>20 c\right)$ (Rani et al. 2015). However, a contradictory result was obtained by Rastorgueva et al. (2011) using data at 1.6, 5, 22, 43, and $86 \mathrm{GHz}$ from 2004: components in the inner jet (to 1 mas from the core) moved rapidly, with speeds up to $\beta_{\text {app }} \approx 20 c$, while the maximum apparent speed observed for components in the outer jet was $10 c$.

Let us consider the results indicated above in our model of a helical jet. According to the 2004 data (Rastorgueva et al. 2011), the position angle of the inner jet was close to its maximum value, $\approx 25^{\circ}$, while the position angle of the outer jet was close to its minimum value, $15^{\circ}$. We found from (2) that the model azimuth angles of the inner and outer jet features are $\varphi_{\text {fast }} \simeq 101^{\circ}$ and $\varphi_{\text {low }} \simeq 259^{\circ}$, respectively. Rani et al. (2015) considered data obtained from September 2008 through October 2010. By this time, the inner components observed in 2004 had moved to the outer part of the jet, and the inner jet is formed by new components whose position angles have their minimum value (see Fig. 8 in Lister et al. 2013). Thus, the azimuthal angles of the inner and outer jet components in $2008-2010$ are $\varphi_{\text {slow }} \simeq 259^{\circ}$ and $\varphi_{\text {fast }} \simeq 101^{\circ}$, respectively. The results of an analysis of the kinematics of the jet components in the interval 1992.72 - 2001.17 indicate that features in the inner jet moved systematically more slowly than those in the outer jet (Bach et al. 2005). Since, as in 2008 - 2010, $\mathrm{PA}_{\text {in }}$ had its minimum value in 1997-1998 (see Fig. 8 in Lister et al. 2013), this result is also consistent with the conclusion that the rapidly moving components observed in both 2004 and in 2008 - 2010 have azimuth angles of $101^{\circ}$, while slowly moving components have azimuth angles of $259^{\circ}$. Note that the physical speed of the components $\beta$ (in units of the speed of light) must be roughly the same in order for the helical structure of the jet to exist over a fairly long time. With a constant $\beta$, the apparent speed

$$
\beta_{\mathrm{app}}=\frac{\beta \sin \theta}{1-\beta \cos \theta}
$$

depends on the angle $\theta$ between the velocity vector of the component and the line of sight. With the indicated azimuth angles, it follows from (5) that, in the case of ballistic motion of components with low and high apparent speeds directed at the same angle to the line of sight, $\theta_{\text {fast }}=\theta_{\text {slow }} \approx 8^{\circ}$. Differences in the apparent speeds must then be due to differences in the physical speeds of the components, which contradicts our assumption that $\beta$ is constant, and we believe that such variations in $\beta$ do not have a natural physical explanation. In the case of non-ballistic motion with a pitch angle of $p \approx 5^{\circ}$, we find from (6)-(13) that the angles to the line of sight for fast and slow components are $\theta_{\text {fast }} \approx 3^{\circ}$ and $\theta_{\text {slow }} \approx 13^{\circ}$, respectively. This can explain the difference in the apparent speeds of components having the maximum and minimum position angles. Consequently, the seemingly contradictory results of the kinematic 


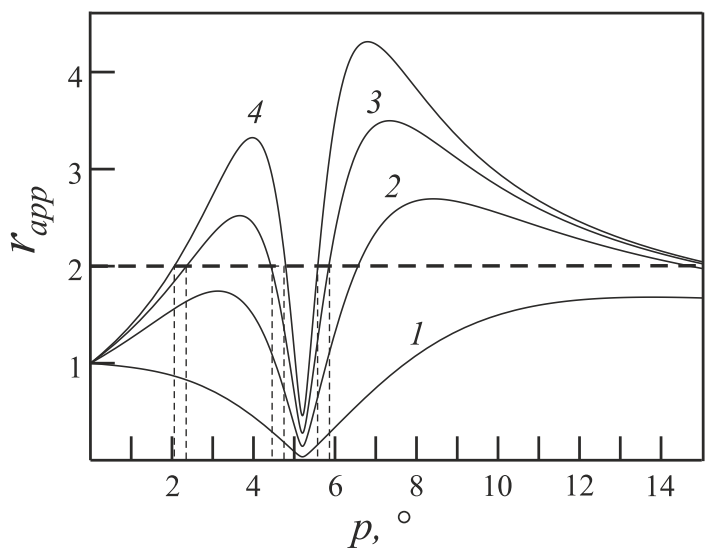

Figure 4. Ratio of the apparent speeds of the outer and inner jet components $r_{\text {app }}$ for $2008-2010$ as a function of the pitch angle p. Plots are shown for $\beta=0.995$ (1), 0.999 (2), 0.9995 (3), and 0.9997 (4) (Lorentz factors of 10, 22, 32, and 41, respectively). The horizontal dashed line shows the observed value of $r_{\text {app }}$, and the values of $p$ for which this can occur are indicated.

analyses of components in the jet of the blazar S5 0716+71 obtained by Rani et al. (2015) and Rastorgueva et al. (2011) can only be explained in a natural way in the case of non-ballistic motions of components, with different azimuthal angles for the inner and outer features at different observing epochs. Note that a number of studies have suggested the existence of non-ballistic trajectories for component motions (see, e.g., Britzen et al. 2009; Rani et al. 2015). However, in this case, $\beta_{\text {app }}$ depends not only on $\varphi$, but also on $p$. Figure reffig:fig4 shows the dependence of the ratio of the apparent speeds of inner and outer jet components $r_{\text {app }}=\beta_{\text {app, out }} / \beta_{\text {app, in }}$ on the pitch angle for epochs 2008-2010, derived from the expression

$$
r_{\text {app }}=\frac{\beta_{\text {app }, \text { out }}}{\beta_{\text {app }, \text { in }}}=\frac{\sin \left(\theta\left(\varphi_{\text {out }}, p\right)\right)\left(1-\beta \cos \theta\left(\varphi_{\text {in }}, p\right)\right)}{\sin \left(\theta\left(\varphi_{\text {in }}, p\right)\right)\left(1-\beta \cos \theta\left(\varphi_{\text {out }}, p\right)\right)},
$$

where $\varphi_{\text {in }}=259^{\circ}$ and $\varphi_{\text {out }}=101^{\circ}$. The observed value of $r_{\text {app }} \approx 2$ (Rani et al. 2015) is possible only if the jet component speed is $\beta>0.999$ (corresponding to a Lorentz factor $\Gamma \gtrsim 22$ ). The pitch angle can be equal to approximately $2^{\circ}, 4.5^{\circ}, 5.5^{\circ}$, and $14^{\circ}$. Substituting these values into (6)-(13), we found that only for $p=5.5^{\circ}$ does the value of $\theta$ vary from $0.5^{\circ}$ to $11^{\circ}$, corresponding to the interval of estimates of $\theta$, obtained from apparent superluminal component motions in the jet (Bach et al. 2005; Rastorgueva et al. 2011; Pushkarev et al. 2009; Savolainen et al. 2010). It follows from (14) that, when $\theta=1^{\circ}-2^{\circ}$ and $\beta=0.999-0.9995$, the apparent speed acquires high values $(20 c-30 c)$, as have been detected in the jet of S5 $0716+71$ (Rani et al. 2015). Using VLBI data, Bach et al. (2005) estimated the minimum Lorentz factor to be $\Gamma=11.6(\beta \approx 0.996)$ and the maximum angle to the line of sight to be $\theta \approx 4.9^{\circ}$. However, they note that a large range of observed apparent component speeds can be realized with $\Gamma>15$ and $\theta<2^{\circ}$, corresponding to Doppler factors $\delta \approx 20-30$. Precisely such high values of $\delta$ are required to explain the high brightness temperatures derived from observations of microvariability of S5 $0716+71$ at centimeter wavelengths (up to $10^{17} \mathrm{~K}$ ) (Bach et al. 2005). Rani et al. (2013) estimated the Doppler factor using several independent methods and obtained the lower limit $\delta \geq 10-20$. In their modeling of the spectral energy distribution in the gamma-ray assuming the action of inverse Compton scattering on the synchrotron photons together with inverse Compton scattering on thermal radiation from hot dust and radiation from the broad-line region, Liao et al. (2014) inferred $\delta$ to be greater than 20. Larionov et al. (2013) explained the photometric and polarization behavior of a flare in S5 0716+71 in October 2011 as due to the motion of a radiating region along a helical path with a pitch angle of $7.5^{\circ}$ for an angle of the jet to the line of sight 5.8 . The long-term trend for increasing brightness of S5 0716+71 from 1994 through 2003 has been interpreted as due to a change in the angle of the jet to the line of sight from $\approx 5^{\circ}$ to $0.7^{\circ}$ with a constant $\Gamma=12$ (corresponding to an increase in $\delta$ from 15 to 22, Nesci et al. 2005). Thus, the estimates of the jet parameters we have obtained are consistent with other independent estimates derived using radio, optical, and gamma-ray observations.

\section{CONCLUSION}

The hypothesis that jets have helical structure has been widely applied. In this case of the blazar S5 0716+71, such structure is suggested by the variations of the position angle of the inner jet, which have a periodic character 
(Bach et al. 2005; Lister et al. 2013). In spite of the fact that the entire interval of VLBI observations of this object encompasses only slightly more than one calculated period of variation of $\mathrm{PA}_{\mathrm{in}}$, we have obtained the geometrical parameters of the helical curve of the jet from the amplitude and period of the variations in $\mathrm{PA}_{\text {in }}$ (Lister et al. 2013), namely, the jet opening angle $\xi=1^{\circ}$ and angle between the axis of the jet helix and the line of sight $\theta_{0}=5.3^{\circ}$. These values are in agreement with analogous quantities found by Pushkarev et al. (2009) based on VLBI maps, and do not depend on whether or not the jet components move along ballistic trajectories. However, with these values of $\theta_{0}$ and $\xi$ and with non-ballistic motions of the components forming the helical shape of the jet, the range of values for the angle $\theta$ between the velocity vectors of the components and the line of sight is appreciably broader than in the case of ballistic motions $-\approx 10^{\circ}$, as opposed to $\approx 2^{\circ}$. This broad range of $\theta$ is manifest through the different estimates of $\theta$ obtained in different studies based on observations at different epochs. Further, $\theta$ can have very small values $\left(-1^{\circ}\right)$, which could result in the high Doppler factors deduced in a number of studies (Montagni et al. 2006; Bach et al. 2005), and also in high apparent speeds for individual components, right up to $37 c$ Rani et al. (2015). It is also possible to explain the differences in apparent component speeds in the inner and outer VLBI jet observed at different epochs (Bach et al. 2005; Rastorgueva et al. 2011) in the case of non-ballistic motions, as well as their different azimuth angles (different orientations of the components relative to the plane containing the line of sight and the axis of the helical curve of the jet). We have used the observed ratio of $\beta_{\text {app }}$ in the outer and inner jet to infer the pitch angle $p=5.5^{\circ}$, at which the components move relative to the ballistic trajectory, and derived a lower limit to the physical speed of the components $\beta>0.999$. Thus, a helical structure of the jet together with non-ballistic motions of its components can explain the wide range of apparent component speeds derived for the inner and outer jet, as well as the wide range of estimates of the angle between the velocity vectors of the jet components and the line of sight.

\section{ACKNOWLEDGMENTS}

The author thanks A.B. Pushkarev for useful comments.

\section{REFERENCES}

Abraham, Z. 2000, A\&A, 355, 915

Abraham, Z., \& Carrara, E. A. 1998, ApJ, 496, 172, doi: 10.1086/305387

Bach, U., Krichbaum, T. P., Ros, E., et al. 2005, A\&A, 433, 815, doi: 10.1051/0004-6361:20040388

Britzen, S., Kam, V. A., Witzel, A., et al. 2009, A\&A, 508, 1205, doi: 10.1051/0004-6361/200810875

Bychkova, V. S., Kardashev, N. S., Boldycheva, A. V., Gnedin, Y. N., \& Maslennikov, K. L. 2006, Astronomy Reports, 50, 802, doi: 10.1134/S1063772906100040

Bychkova, V. S., Vol'vach, A. E., Kardashev, N. S., et al. 2015, Astronomy Reports, 59, 851, doi: 10.1134/S1063772915080016

Camenzind, M., \& Krockenberger, M. 1992, A\&A, 255, 59

Caproni, A., \& Abraham, Z. 2004, MNRAS, 349, 1218, doi: 10.1111/j.1365-2966.2004.07550.x

Caproni, A., Abraham, Z., \& Monteiro, H. 2013, MNRAS, 428, 280, doi: 10.1093/mnras/sts014

Dai, Y., Wu, J., Zhu, Z.-H., et al. 2013, ApJS, 204, 22, doi: 10.1088/0067-0049/204/2/22

Gabuzda, D. C., Pushkarev, A. B., \& Cawthorne, T. V. 2000, MNRAS, 319, 1109, doi: $10.1046 /$ j.1365-8711.2000.03932.x
Gorshkov, A. G., Ipatov, A. V., Konnikova, V. K., et al. 2011a, Astronomy Reports, 55, 97, doi: 10.1134/S106377291102003X

—. 2011b, Astronomy Reports, 55, 1096, doi: 10.1134/S1063772911120043

Hardee, P. E., \& Norman, M. L. 1988, ApJ, 334, 70, doi: $10.1086 / 166818$

Larionov, V. M., Jorstad, S. G., Marscher, A. P., et al. 2013, ApJ, 768, 40, doi: 10.1088/0004-637X/768/1/40

Liao, N. H., Bai, J. M., Liu, H. T., et al. 2014, ApJ, 783, 83, doi: 10.1088/0004-637X/783/2/83

Lister, M. L., Aller, M. F., Aller, H. D., et al. 2013, AJ, 146, 120, doi: 10.1088/0004-6256/146/5/120

Meier, D. L., Koide, S., \& Uchida, Y. 2001, Science, 291, 84, doi: 10.1126/science.291.5501.84

Montagni, F., Maselli, A., Massaro, E., et al. 2006, A\&A, 451, 435, doi: 10.1051/0004-6361:20053874

Nesci, R., Massaro, E., Rossi, C., et al. 2005, AJ, 130, 1466, doi: $10.1086 / 444538$

Poon, H., Fan, J. H., \& Fu, J. N. 2009, ApJS, 185, 511, doi: 10.1088/0067-0049/185/2/511 
Pushkarev, A. B., Kovalev, Y. Y., Lister, M. L., \& Savolainen, T. 2009, A\&A, 507, L33, doi: 10.1051/0004-6361/200913422

Rani, B., Krichbaum, T. P., Marscher, A. P., et al. 2015, A\&A, 578, A123, doi: 10.1051/0004-6361/201525608

Rani, B., Krichbaum, T. P., Fuhrmann, L., et al. 2013, A\&A, 552, A11, doi: 10.1051/0004-6361/201321058

Rastorgueva, E. A., Wiik, K. J., Bajkova, A. T., et al. 2011, A\&A, 529, A2, doi: 10.1051/0004-6361/201015241

Savolainen, T., Homan, D. C., Hovatta, T., et al. 2010, A\&A, 512, A24, doi: 10.1051/0004-6361/200913740
Sbarufatti, B., Treves, A., \& Falomo, R. 2005, ApJ, 635, 173, doi: 10.1086/497022

Villata, M., \& Raiteri, C. M. 1999, A\&A, 347, 30

Vol'Vach, A. E., Larionov, M. G., Kardashev, N. S., et al. 2009, Astronomy Reports, 53, 777, doi: 10.1134/S1063772909090017

Volvach, A. E., Volvach, L. N., Bychkova, V. S., et al. 2012, Astronomy Reports, 56, 275, doi: 10.1134/S1063772912030079

Wagner, S. J., Witzel, A., Heidt, J., et al. 1996, AJ, 111, 2187, doi: $10.1086 / 117954$ 